\title{
PERTURBATIVE RENORMALIZATIONS OF ANYON QUANTUM MECHANICS用
}

\author{
Giovanni AMELINO-CAMELIA \\ Center for Theoretical Physics \\ Laboratory for Nuclear Science \\ and Department of Physics \\ Massachusetts Institute of Technology \\ Cambridge, Massachusetts 02139 USA
}

\begin{abstract}
In bosonic end perturbative calculations for quantum mechanical anyon systems a regularization and renormalization procedure, analogous to those used in field theory, is necessary. I examine the reliability and the physical interpretation of the most commonly used bosonic end regularization procedures. I then use the regularization procedure with the most transparent physical interpretation to derive some bosonic end perturbation theory results on anyon spectra, including a 3-anyon ground state energy.
\end{abstract}

Submitted to: Physical Review D

MIT-CTP-2321

June, 1994

*This work is supported in part by funds provided by the U.S. Department of Energy (D.O.E.) under cooperative agreement \#DE-FC02-94ER40818, and by the Istituto Nazionale di Fisica Nucleare (INFN, Frascati, Italy). 


\section{Introduction}

In $2+1$ dimensions the rotation group is abelian $(S O(2))$, and as a consequence, besides bosons and fermions, also particles with neither integer nor half-integer spin, anyons, can exist [1]. Free anyons can be described [2], in what is called "boson gauge"

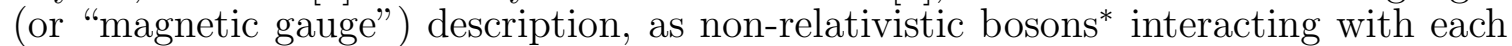
other through the mediation of an abelian Chern-Simons gauge field; the corresponding Hamiltonian is

$$
\begin{gathered}
H_{b}=\frac{1}{2 m} \sum_{n}\left(\mathbf{p}_{n}-\nu \mathbf{a}_{n}\right)^{2}, \\
a_{n}^{k} \equiv-\epsilon^{k j} \sum_{m(\neq n)} \frac{r_{n}^{j}-r_{m}^{j}}{\left|\mathbf{r}_{n}-\mathbf{r}_{m}\right|^{2}},
\end{gathered}
$$

where $\mathbf{r}_{\mathbf{n}} \equiv\left(r_{n}^{1}, r_{n}^{2}\right)$ is the position vector of the n-th particle.

An alternative description of anyons, called "anyon gauge" description, can be derived from the boson gauge using the following transformation

$$
\Psi_{b} \rightarrow \Psi_{a}=U \Psi_{b}, \quad H_{b} \rightarrow H_{a}=U H_{b} U^{-1}
$$

where

$$
U \equiv \exp \left[-i \nu \sum_{m \neq n} \theta_{n m}\right]
$$

the $\Psi_{b}$ 's are the bosonic wave functions that appear in the boson gauge description, and $\theta_{n m}$ are the azimuthal angles of the relative vectors $\mathbf{r}_{n}-\mathbf{r}_{m}$. It is easy to verify that $H_{a}$ has the form $\sum_{n} \mathbf{p}_{n}^{2} / 2 m$ of an ordinary free Hamiltonian; moreover, since the boson gauge wave functions $\Psi_{b}$ are single-valued and $U$ is not single-valued, the $\Psi_{a}$ 's are multi-valued. Indeed, with the transformation (1.3) one removes the interaction by redefining (in a multivalued fashion) the phase of the wave functions, a procedure which is made possible by the fact that $\mathbf{a}_{\mathbf{n}}$ is a (singular) pure gauge potential[2]. The multi-valuedness of the $\Psi_{a}$ 's is directly related to the anomalous quantum statistics of anyons, which, in particular, prescribes[四] that the quantum mechanical wave functions describing the relative motion of two anyons in polar coordinatest], satisfy the following condition

$$
\Psi_{a}(r, \theta+\pi)=e^{-i \nu \pi} \Psi_{a}(r, \theta)
$$

It is easy to verify that the $\Psi_{a}$ 's defined in Eq.(1.3) satisfy the condition (1.5).

*Indeed, in 2+1 dimensions particles with any statistics can be described as bosons interacting through an appropriate statistical interaction.

${ }^{\dagger}$ Here the relative angle runs from $-\infty$ to $\infty$, without identifying angles which differ by multiples of $2 \pi$, so that it keeps track of the number of windings [1]. 
The parameter $\nu$, called "statistical parameter", characterizes the type of anyons, i.e. their statistics. In particular, from Eq.(1.5) one realizes that anyons with even (odd) integer $\nu$ verify bosonic (fermionic) statistics, whereas the noninteger values of $\nu$ correspond to particles with statistics interpolating between the bosonic and the fermionic case. Without any loss of generality [1], I shall restrict the values of $\nu$ to be in the interval $[-1,1]$.

Whereas in the case of bosons and fermions the wave functions for $N$ noninteracting particles or $N$ particles interacting via a separable Hamiltonian (i.e. an Hamiltonian which can be written as a sum of corresponding one-particle Hamiltonians) are simply given by appropriately symmetrized products of one-particle wave functions, in the case of general-type anyons, because of the anomalous statistics, these factorizations do not occur, rendering the study of any $N$-anyon problem extremely difficult. It is essentially for this reason that the problem of finding all the exact energy eigenvalues and eigenfunctions, even for very simple anyon systems, is still unsolved for $N>2$.

The realization that anyons are relevant to the understanding of some condensed matter phenomena, most notably the fractional quantum Hall effect[3], has motivated numerous recent studies of the $N$-anyon problem. In particular, the $N$-identicalanyon in an external harmonic potential system has played a central role in these recent investigations. The harmonic potential, by discretizing the spectrum, allows to disentangle the dependence on the statistical parameter $\nu$; moreover, the virial coefficients can be deduced from the solutions of the harmonic potential problem by taking in appropriate fashion the limit of vanishing oscillator frequency [5, 6].

The $N$-anyon in harmonic oscillator problem has been completely solved 11 for $N=2$, but only an incomplete set of exact eigensolutions has been found [7, 8, 9] for $N>2$. Interestingly all the known eigenenergies depend linearly on the statistical parameter $\nu$.

Besides the search for exact solutions, there has been much effort in the perturbative[5,6,9-16] and in the numerical [17, 18] study of the $N$-anyon problem. Both the perturbative and the numerical studies have led to interesting results; most notably, they have shown that there are energies with nonlinear dependence on $\nu$ among those that are not presently known exactly.

In the proposed perturbative approaches, called "bosonic end" and "fermionic end" perturbative approaches, one studies either "quasi-bosonic" anyons (i.e. anyons with small $\nu$, whose anomalous statistics is close to being bosonic) or "quasi-fermionic" anyons, by using a perturbative expansion in which the small parameter is the deviation of the statistics (indicated by the statistical parameter $\nu$ ) from the bosonic or the fermionic limit.

The analysis presented in this paper concerns the bosonic end perturbative approach, which, because of the non-analyticity[1, 19] of the limit $\nu \rightarrow 0$, is afflicted by some spurious divergencies, and therefore requires regularization[9,11,16,19-21]. In order to see the mechanism that leads to these divergencies, let us look at the simple case of two anyons in an harmonic oscillator potential; the boson gauge Hamiltonian

\footnotetext{
${ }^{\ddagger}$ Note that the harmonic oscillator potential has the same form as one of the generators of the $S O(2,1)$ symmetry transformations of the system[ [4. It would be interesting to investigate whether this fact plays a role in the various uses made of the harmonic oscillator potential in the study of anyon quantum mechanics, especially concerning the limiting procedure to be followed in deriving the virial coefficients.
} 
that describes the relative motion is

$$
H_{2}=-\frac{1}{r} \partial_{r}\left(r \partial_{r}\right)-\frac{1}{r^{2}} \partial_{\phi}^{2}+r^{2}-\frac{2 i \nu}{r^{2}} \partial_{\phi}+\frac{\nu^{2}}{r^{2}}
$$

In the perturbative calculations about $\nu=0$, one runs into an inconsistency because the matrix elements of $\frac{\nu^{2}}{r^{2}}$ between 0 -th order in $\nu$ (i.e. bosonic) s-wave functions are logarithmically divergent; for example, to the second order in $\nu$, one of the contributions to the ground state energy is given by

$$
\begin{aligned}
<\Omega_{\text {boson }}\left|\frac{\nu^{2}}{r^{2}}\right| \Omega_{\text {boson }}> & =\int_{0}^{\infty} \int_{0}^{2 \pi} r d r d \phi \frac{e^{-\frac{r^{2}}{2}}}{\pi^{\frac{1}{2}}} \frac{\nu^{2}}{r^{2}} \frac{e^{-\frac{r^{2}}{2}}}{\pi^{\frac{1}{2}}} \\
& =2 \nu^{2} \int_{0}^{\infty} \frac{\exp \left(-r^{2}\right)}{r} d r \sim \infty
\end{aligned}
$$

Many of the results presently available on the unknown portion of the anyon spectra, have been obtained using bosonic end perturbation theory. However, some of the regularization procedures used in these calculations require rather arbitrary manipulations. Only recently, motivated by the results of some related field theory analysis 222, a regularization procedure with a clearer physical interpretation has been proposed [16, 20, 21]. This regularization procedure is based on the introduction of a repulsive $\delta$-function potential, and I shall refer to it as the " $\delta$-function regularization".

The plan of this paper is the following. In the next section, I review the three most commonly used procedures of regularization of bosonic end perturbation theory. In particular, this allows me to show that, among these procedures, the $\delta$-function regularization is indeed the one with the clearest physical interpretation. In Sec.III, I discuss the relations among the different regularization procedures. In Sec.IV, I explicitly test the reliability of the $\delta$-function regularization by using it in rederiving several results that have already been obtained exactly or numerically. Finally, Sec.V is devoted to my conclusions.

\section{Regularizations of Bosonic End Perturbation The- ory}

In this section I briefly review the three most commonly used procedures of regularization of bosonic end perturbation theory. In order to be specific, I limit the discussion to the case of 2 identical anyons in an external harmonic oscillator potential, whose

\footnotetext{
$\S$ A fourth regularization procedure has been discussed in Ref. 21]; however, that regularization procedure has not been used in the study of anyon spectra, and in Ref.21 it was shown to be completely equivalent to one of the three regularization procedures discussed in the following.

\Most of these tests have already been performed in the short publication [16]; in Sec.IV I review them, giving additional details on the calculations.
} 
relative motion is described by the Hamiltonian $H_{2}$ in Eq.(1.6). As I mentioned, the exact solutions of the $H_{2}$-eigenproblem are known; they are given by [1]

$$
\begin{gathered}
E_{n, l, \nu}^{\text {exact }}=(4 n+2|l+\nu|+2), \\
\mid \Psi_{n, l, \nu}^{\text {exact }}>=N_{n, l}^{\nu} r^{|l+\nu|} e^{-\frac{r^{2}}{2}+i l \phi} L_{n}^{|l+\nu|}\left(r^{2}\right) .
\end{gathered}
$$

where the $L_{n}^{x}$ are Laguerre polynomials, and the $N_{n, l}^{\nu}$ are normalization constants.

\subsection{Regularization Method I}

It has been proposed [11, 12 that, in order to obtain a consistent bosonic end perturbative approach, one should not apply perturbation theory to the original eigenproblem $H_{N}|\Psi>=E| \Psi>$ (where $H_{N}$ is a $N$-anyon Hamiltonian), but rather to the modified eigenproblem $\tilde{H}_{N}|\tilde{\Psi}>=E| \tilde{\Psi}>$ where $\tilde{H}_{N}^{\nu}$ and $\mid \tilde{\Psi}>$ are related to the original $H_{N}$ and $\mid \Psi>$ by

$$
\begin{gathered}
\left|\tilde{\Psi}>=\left(\prod_{n<m}\left(\mathbf{r}_{\mathbf{n}}-\mathbf{r}_{\mathbf{m}}\right)^{-|\nu|}\right)\right| \Psi> \\
\tilde{H}_{N}^{\nu}=\left(\prod_{n<m}\left(\mathbf{r}_{\mathbf{n}}-\mathbf{r}_{\mathbf{m}}\right)^{-|\nu|}\right) H_{N}\left(\prod_{n<m}\left(\mathbf{r}_{\mathbf{n}}-\mathbf{r}_{\mathbf{m}}\right)^{|\nu|}\right) .
\end{gathered}
$$

In the case of two anyons in an external harmonic oscillator potential, from (1.6) one finds that the Hamiltonian is

$$
\tilde{H}_{2}^{\nu}=r^{-|\nu|} H_{2} r^{|\nu|}=\frac{1}{r} \partial_{r}\left(r \partial_{r}\right)-\frac{1}{r^{2}} \partial_{\phi}^{2}+r^{2}-\frac{2 i \nu}{r^{2}} \partial_{\phi}-\frac{2|\nu|}{r} \partial_{r}
$$

The exact solutions for the $\tilde{H}_{2}^{\nu}$-eigenproblem are easily found from (2.1) and (2.2):

$$
\begin{gathered}
\tilde{E}_{n, l, \nu}^{\text {exact }}=E_{n, l, \nu}^{\text {exact }}=(4 n+2|l+\nu|+2) \\
\left|\tilde{\Psi}_{n, l, \nu}^{\text {exact }}>=\frac{\tilde{N}_{n, l}^{\nu}}{N_{n, l}^{\nu}} r^{-|\nu|}\right| \Psi_{n, l, \nu}^{\text {exact }}>=\tilde{N}_{n, l}^{\nu} r^{|l+\nu|-|\nu|} \exp \left(-\frac{r^{2}}{2}+i l \phi\right) L_{n}^{|l+\nu|}\left(r^{2}\right)
\end{gathered}
$$

where $\tilde{N}_{n, l}^{\nu}$ are normalization constants.

Notice that: (i) $\tilde{H}_{2}^{\nu}$ is not Hermitian, (ii) the transformation $|\Psi>\rightarrow| \tilde{\Psi}>\sim$ $r^{-|\nu|} \mid \Psi>$ is not unitary, (iii) $\tilde{H}_{2}^{\nu}$ depends non-analytically on $\nu$. Moreover, the eigensolutions (i.e. the eigenfunctions and the eigenvalues) of $\tilde{H}_{2}^{\nu}$ are non-smooth 
functions of $\nu$ for every value of $l$, whereas the $H_{2}$-eigensolutions are non-smooth only for $l=0$.

Although the exact $H_{2}$-eigenproblem and $\tilde{H}_{2}^{\nu}$-eigenproblem are simply related, the same is not true when perturbation theory is used. In particular, the non-analytic dependence on $\nu$ present in $\tilde{H}_{2}^{\nu}$ is such to compensate (in perturbation theory) for the other non-analitycities present in the problem, and, as a consequence, the bosonic end perturbation theory for the $\tilde{H}_{2}^{\nu}$-eigenproblem is not affected by divergencies [11, 19.

The "regularization method I" consists in using the (finite) results of perturbation theory applied to the $\tilde{H}_{N}^{\nu}$-eigenproblem to derive, based on the relation between $\mid \tilde{\Psi}>$ and $|\Psi\rangle$, the corresponding results for the original $H_{N}$-eigenproblem.

This regularization method I has undergone numerous tests [6, 11, 19. For the 2 -anyon in harmonic potential problem all the regularization method I results have been shown to be consistent with the exact solutions. Moreover, several first order few-anyon eigenenergies have been calculated and the results are in agreement with the expansion in $\nu$ of the exact solutions.

Unfortunately, the physics behind the manipulations involved in the regularization method $\mathrm{I}$ is not completely understood. For example, it is not clear for what reason among the Hamiltonians $\tilde{H}_{2}^{\beta} \equiv r^{-|\beta|} H_{2} r^{|\beta|}$ only the one with $\beta=\nu$ leads to the correct anyon spectra. It would also be interesting to comprehend the role in the regularization procedure of the fact that the transformation $|\Psi\rangle \rightarrow|\tilde{\Psi}\rangle$ is singular at $r=0$ and non-unitary, and that $\tilde{H}_{N}^{\nu}$ is not Hermitian. An "hermitized version" of the regularization method I, in which the perturbation theory is based on the Hermitian Hamiltonian $\left(\tilde{H}_{N}^{\nu+}+\tilde{H}_{N}^{\nu}\right) / 2$, has also been considered in the literature [12, [15], but it can be used only for rather limited tasks [12, 19] (more on this in Sec.III).

\subsection{Regularization Method II}

Another perturbative approach to the study of quasi-bosonic anyons used in the literature 9, 13, 14] is based on the idea that, if one wants to describe perturbatively the conventional "non-colliding" anyons 7 in terms of (0-th order) bosonic wave functions, one needs to modify the bosonic wave functions so that they have an appropriate hard core. Specifically, one substitutes the bosonic wave functions $\left|\Psi^{(0)}\right\rangle$ with "regularized bosonic wave functions" $\mid \Psi^{(0) R}>$ in all the divergent matrix elements of the naive perturbative approach (like the matrix element in Eq.(1.7)). For the 2-body case (the generalization to the N-body case is given in the references) the $\mid \Psi^{(0) R}>$ are defined by

$$
\left|\Psi_{n, l}^{(0) R \frac{\nu}{2}}>\equiv N_{n, l}^{R \nu} r^{\frac{|\nu|}{2}}\right| \Psi_{n, l}^{(0)}>
$$

where $N_{n, l}^{R \nu}$ are normalization constants. Eq.(2.8) indeed implements a hard core condition; in fact $\left|\Psi_{n, l}^{(0) R \frac{\nu}{2}}\right\rangle=0$ for $r=0$. As a consequence, substituting the

* "Non-colliding anyons" are anyons whose wave functions vanish at the points of overlap. The possibility of "colliding" anyons has also been examined in the literature (for example this subject is discussed in Ref. [23]), but in this paper only the conventional "non-colliding" anyons are considered. 
$\mid \Psi_{n, l}^{(0) R \frac{\nu}{2}}>$ to the $\mid \Psi_{n, l}^{(0)}>$ in the divergent matrix elements of the naive perturbative approach, one obtains finite matrix elements; for example the matrix element in (1.7) is substituted by

$$
\begin{aligned}
<\Psi_{0,0}^{(0) R \frac{\nu}{2}}\left|\frac{\nu^{2}}{r^{2}}\right| \Psi_{0,0}^{(0) R \frac{\nu}{2}}> & =\int_{0}^{\infty} \int_{0}^{2 \pi} \frac{r d r d \phi}{\pi \Gamma\left(1+\frac{|\nu|}{2}\right)} \frac{\nu^{2}}{r^{2}} e^{-r^{2}} r^{|\nu|} \\
& =\nu^{2} \frac{\Gamma\left(\frac{|\nu|}{2}\right)}{\Gamma\left(1+\frac{|\nu|}{2}\right)}=2|\nu| .
\end{aligned}
$$

The choice of the "regularizing exponent" $\frac{|\nu|}{2}$ in (2.8), is only justified a posteriori by the agreement of the perturbative results with the exact results [19].

It is important to notice that, although (2.8) and (2.7) are formally similar, this "regularization method II" and the regularization method I represent conceptually different approaches. In the regularization method I, one studies a different eigenproblem, since the Hamiltonian $\tilde{H}_{N}$ is different from $H_{N}$, and perturbation theory is applied to the eigenproblem of $\tilde{H}_{N}$; only at the end one recovers a perturbative result for the original eigenproblem through appropriate conversion formulas [19]. In the regularization method II, equation (2.8), which involves only the 0 -th order wave functions, effectively defines regularized matrix elements for the original theory, and no equivalent eigenproblem is introduced; in the calculations one uses the original Hamiltonian, and the results refer directly (no conversion formulas are needed) to the original eigensolutions.

Another important observation is that in Eq.(2.9) a matrix element apparently of order $\nu^{2}$ actually gives a contribution of order $\nu$. This is a general aspect of the regularization method $\mathrm{I}$, and is due to the fact that near $\nu=0$ some matrix elements $<\Psi_{0}^{R \frac{\nu}{2}}\left|r^{-2}\right| \Psi_{0}^{R \frac{\nu}{2}}>$ have a pole $\frac{1}{|\nu|}$ which is a remainder of the original divergencies. As a consequence, the complete perturbative result of order $\nu^{n}$ requires the evaluations of terms which in ordinary perturbation theory appear at any order $\nu^{m}$, with $n \leq m \leq 2 n$.

In spite of these peculiarities, also the regularization method II has proven very reliable in a series of tests. The first order eigenenergies and eigenfunctions for the 2-anyon Hamiltonian $\mathrm{H}_{2}$ have been calculated 19 and the results are in agreement with the expansion in $\nu$ of the exact solutions. Some 3 and 4 anyon eigenenergies have been calculated 99, 13, 14 to second order in $\nu$, with results in agreement with the numerical solutions obtained in Refs. 17, 18 and (for the states for which such exact solutions are available) with the exact solutions found in Refs. [7, 8, 9]. However, in the second order calculations some spurious infinities must be neglected in order to obtain the correct results [14. Even though this further regularization can be cast into a general procedure 14 (the same term is neglected in all second order calculations), this is another arbitrary manipulation required by the regularization method II.

The quantitative successes of the regularization method II are surprising considering the apparent arbitrariness of some of the manipulations involved. In particular, even if the basic physical idea (that in order to study conventional anyons the unperturbed wave functions must have an hard-core) should be correct, one would like to understand why the definition (2.8) is the right one. For example, if one used the definition $\left|\Psi_{0}^{R \alpha}>\equiv N_{\Psi_{0}, \alpha} r^{|\alpha|}\right| \Psi_{0}>$ the $\mid \Psi_{0}^{R \alpha}>$ 's would have an hard-core independently of the value of $\alpha$, and it is not clear for what reason the choice $\alpha=\nu / 2$ is the only one leading to the correct anyon spectra [9, 19]. 


\section{$2.3 \quad \delta$-function regularization}

For the conventional non-colliding anyons the wave functions vanish at the points of overlap, and the addition of a repulsive $\delta$-function potential to the Hamiltonian $H_{N}$ of a quantum mechanical $N$-anyon system has no physical consequences (see for example Ref. 24]), i.e. the exact eigensolutions are unaffected by it. One can therefore apply small- $\nu$ perturbation theory, instead of to the original Hamiltonian $H_{N}$, to the equivalent Hamiltonian $H_{N}^{\delta}$, given by

$$
H_{N}^{\delta} \equiv H_{N}+2 \pi|\nu| \sum_{m<n} \delta^{(2)}\left(\mathbf{r}_{\mathbf{n}}-\mathbf{r}_{\mathbf{m}}\right)
$$

For our 2-anyon in harmonic potential problem the "regularized Hamiltonian" is

$$
H_{2}^{\delta} \equiv-\frac{1}{r} \partial_{r}\left(r \partial_{r}\right)-\frac{1}{r^{2}} \partial_{\phi}^{2}+r^{2}-\frac{2 i \nu}{r^{2}} \partial_{\phi}+2 \pi|\nu| \delta^{(2)}(\mathbf{r})+\frac{\nu^{2}}{r^{2}}=H_{2}+2 \pi|\nu| \delta^{(2)}(\mathbf{r})
$$

Although the (exact) $H_{2}^{\delta}$-eigenproblem is completely equivalent to the $H_{2}$-eigenproblem (they have precisely the same eigensolutions), $H_{2}^{\delta}$ is more suitable for perturbation theory; in fact, the added $\delta$-function potential leads to divergencies which exactly cancel those introduced by the $\nu^{2} / r^{2}$ term, rendering finite the results of bosonic end perturbation theory [16].

I shall illustrate the mechanism that leads to these cancellations in Sec.IV; here I want to discuss the physical interpretation of the $\delta$-function regularization procedure. Within the context of perturbation theory in quantum mechanics, the addition to the original Hamiltonian of a term $2 \pi|\nu| \delta^{(2)}(\mathbf{r})$ can simply be interpreted as an expedient to implement the hard core boundary conditions in the perturbative calculations[?. One can also see, following the analysis in Refs. [20, 26], that such a $\delta$-function potential is naturally induced by a procedure of perturbative renormalization of anyon quantum mechanics, in complete analogy with the structure of the regularization and renormalization procedures used in field theory.

Indeed, in the study of the non-relativistic field theories that correspond to our quantum mechanical problem it has been shown 22, 25] that a quartic contact interaction (the field theoretical analog of a $\delta$-function potential) is necessary for renormalization. In these field theory contexts one can also see that at some critical values of the quartic contact interaction strength the theory is finite (and preserves classical conformal invariance). In the framework of a perturbative renormalization of anyon quantum mechanics a similar interpretation can be given of the choicef $2 \pi|\nu|$ for the coefficient of the $\delta$-function term which is necessary in order to reproduce the exact results. The critical value $2 \pi|\nu|$ can be shown 25] to be the one that implements the boundary conditions appropriate for the conventional non-colliding anyons in the perturbation theory.

\footnotetext{
${ }^{\dagger}$ More on contact interactions and boundary conditions can be found in Refs. [26, 27.

$\ddagger$ Note, however, that both in field theory and in quantum mechanics these critical values of the interaction strengths are only determined up to sign ambiguities (see for example Ref.[25]), and the sign-choice can only be made by comparing perturbative results with the exact results one wants to reproduce.
} 
In summary, our $\delta$-function regularization procedure has indeed a rather transparent physical interpretation, and makes contact with other interesting problems of theoretical physics.

\section{Relations between Regularization Procedures}

In this section I will individuate some relations between the regularization procedures discussed in the preceding section. Again for definiteness and simplicity my discussion is specialized to the 2-anyon in external harmonic oscillator potential problem.

Let me start by looking at the relation between the regularization method I and the $\delta$-function regularization. This is most simply seen by observing that 12

$$
\frac{\tilde{H}_{2}^{\beta}+\tilde{H}_{2}^{\beta+}}{2} \sim-\frac{1}{r} \partial_{r}\left(r \partial_{r}\right)-\frac{1}{r^{2}} \partial_{\phi}^{2}+r^{2}-\frac{2 i \nu}{r^{2}} \partial_{\phi}+2 \pi|\beta| \delta^{(2)}(\mathbf{r})
$$

for $\beta=\nu$ includes the regulating $\delta$-function potential $2 \pi|\nu| \delta^{(2)}(\mathbf{r})$. This suggests that the regularization method I might be equivalent to the $\delta$-function regularization. Indeed, following the analysis of Ref. [21] one finds that $H_{2}^{\delta}$ can be obtained from $\tilde{H}_{2}^{\nu}$ by performing a non-unitary transformation that is the inverse of the ones used in Eq.(2.5), i.e.

$$
H_{2}^{\delta} \sim r^{|\nu|} \tilde{H}_{2}^{\nu} r^{-|\nu|}
$$

It is therefore not surprising that the bosonic end perturbative approaches based on $H_{2}^{\delta}$ and $\tilde{H}_{2}^{\nu}$ lead to consistent results, as indicated by the calculations in the literature.

There is one more noteworthy observation to be made about Eq.(3.1). In fact, this equation shows that (due to the omission of the important $\nu^{2} / r^{2}$ term) a bosonic end perturbation theory based on the Hamiltonian $\left(\tilde{H}_{2}^{\nu}+\tilde{H}_{2}^{\nu+}\right) / 2$, first discussed in Ref. [12], is not equivalent to the $\delta$-function regularized bosonic end perturbation theory. As already noticed in Ref. [12], the Hamiltonian $\left(\tilde{H}_{2}^{\nu}+\tilde{H}_{2}^{\nu+}\right) / 2$ can only be used to obtain first order results (as shown in Ref. [19], the second order results are divergent).

Concerning the relation between the $\delta$-function regularization and the regularization method II, I observe that

$$
\left.<\Psi_{m, k}^{(0)}|2 \pi| \nu\left|\delta^{(2)}(\mathbf{r})\right| \Psi_{n, l}^{(0)}\right\rangle=\left[<\Psi_{m, k}^{(0) R \frac{\nu}{2}}\left|\frac{\nu^{2}}{r^{2}}\right| \Psi_{n, l}^{(0) R \frac{\nu}{2}}>\right]_{O(\nu)}
$$

This suggests that the regularization of the wave functions $\left|\Psi_{n, l}^{(0)}>\rightarrow\right| \Psi_{n, l}^{(0) R \frac{\nu}{2}}>$ used in the regularization method II is in some correspondence with the regularization of the Hamiltonian $H_{2} \rightarrow H_{2}+2 \pi|\nu| \delta^{(2)}(\mathbf{r})$ used in the $\delta$-function regularization. However, it is easy to see 14 that (unlike the second order results of the $\delta$-function regularization) the second order results of the regularization method II require additional regularization. (As I mentioned, this additional regularization can be cast into 
a general procedure that has been shown to give correct second order results in several tests [9, 13, 14], but presently the situation of the higher orders in the perturbative expansion is not clear.) In fact, one can easily see that the regularization method II is closer in spirit* to the "hermitized version" of the regularization method I, but is formulated in a way that naturally suggests a general procedure of regularization of the second order results.

\section{Tests of the $\delta$-function Regularization}

I now perform some direct tests of the reliability of the $\delta$-function regularization. I calculate some first and second order eigenenergies and some first order eigenfunctions, and compare them with the corresponding exact results. I consider identical anyons in an external harmonic oscillator potential; however, it is easy to realize that the particular form of the potential does not play a role in the regularization procedure. Indeed, the divergence that one is regularizing results from the "free-anyon part" of the Hamiltonian.

\section{$4.1 \quad 2$-anyon energies and wave functions}

As a first direct test of its reliability, in this subsection I use the $\delta$-function regularization in the evaluation of the first and second order eigenenergies and the first order eigenfunctions of the 2-anyon Hamiltonian $\mathrm{H}_{2}$ given in Eq.(1.6), whose $\delta$-function regularized Hamiltonian $H_{2}^{\delta}$ was given in Eq.(2.11). My analysis is limited to the anyonic states whose $\nu \rightarrow 0$ limit are bosonic states with angular momentum $l=0$. For the states with $l \neq 0$ no divergence is present to begin with [9, 11, 19], and the consistency of the $\delta$-function regularization can be verified in complete analogy with the corresponding results obtained for the other procedures. [N.B. The $\delta$-function potential does not contribute to the matrix elements involving unperturbed states with $l \neq 0$, because these states vanish for $\mathbf{r}=0$.]

Concerning the first order energies, one easily finds

$$
\begin{array}{r}
E_{n, 0, \nu}^{(1)}=<\Psi_{n, 0}^{(0)}\left|-\frac{2 i \nu}{r^{2}} \partial_{\phi}+2 \pi\right| \nu\left|\delta^{(2)}(\mathbf{r})\right| \Psi_{n, 0}^{(0)}> \\
=<\Psi_{n, 0}^{(0)}|2 \pi| \nu\left|\delta^{(2)}(\mathbf{r})\right| \Psi_{n, 0}^{(0)}>=2|\nu|,
\end{array}
$$

which is clearly in agreement with Eq.(2.1).

The first order eigenfunctions are given by

$$
\left|\Psi_{n, 0, \nu}^{(1)}>=\sum_{m, l \neq n, 0} \frac{<\Psi_{m, l}^{(0)}\left|-\frac{2 i \nu}{r^{2}} \partial_{\phi}+2 \pi\right| \nu\left|\delta^{(2)}(\mathbf{r})\right| \Psi_{n, 0}^{(0)}>}{E_{n, 0}^{(0)}-E_{m, l}^{(0)}}\right| \Psi_{m, l}^{(0)}>
$$

*As shown by Eq.(3.3) the matrix elements $<\Psi_{m, k}^{(0) R \frac{\nu}{2}}\left|\frac{\nu^{2}}{r^{2}}\right| \Psi_{n, l}^{(0) R \frac{\nu}{2}}>$ of the regularization method II correspond to the matrix elements $<\Psi_{m, k}^{(0)}|2 \pi| \nu\left|\delta^{(2)}(\mathbf{r})\right| \Psi_{n, l}^{(0)}>$ of the hermitized version of the regularization method I, and in both these approaches one essentially misses second order contributions of the type $<\Psi_{m, k}^{(0)}\left|\frac{\nu^{2}}{r^{2}}\right| \Psi_{n, l}^{(0)}>$. 


$$
\begin{aligned}
& =\sum_{m[\neq n]} \frac{<\Psi_{m, 0}^{(0)}|2 \pi| \nu\left|\delta^{(2)}(\mathbf{r})\right| \Psi_{n, 0}^{(0)}>}{E_{n, 0}^{(0)}-E_{m, 0}^{(0)}} \mid \Psi_{m, 0}^{(0)}> \\
& =-\frac{|\nu|}{2 \sqrt{\pi}} \sum_{m \neq n} \frac{L_{m}^{0}\left(r^{2}\right)}{m-n} e^{-\frac{r^{2}}{2}} .
\end{aligned}
$$

Using properties of the Laguerre polynomials one can verify (with some algebra) that the result (4.2) is in agreement with Eq.(2.2). For example one obtains

$$
\mid \Psi_{2,0, \nu}^{(1)}>=\frac{|\nu|}{\sqrt{\pi}} e^{-\frac{r^{2}}{2}}\left[\frac{3}{2}-r^{2}+\frac{1}{4}(2 \gamma-3+4 \ln (r)) L_{2}^{0}\left(r^{2}\right)\right]
$$

which agrees with the first order term in the expansion in $\nu$ of $\left|\Psi_{2,0, \nu}^{\text {exact }}\right\rangle$.

From (2.11) one sees that the second order energies are given by

$$
\begin{aligned}
& E_{n, 0, \nu}^{(2)}=E_{n, 0, \nu}^{(2, a)}+E_{n, 0, \nu}^{(2, b)} \\
& E_{n, 0, \nu}^{(2, a)} \equiv<\Psi_{n, 0}^{(0)}\left|\frac{\nu^{2}}{r^{2}}\right| \Psi_{n, 0}^{(0)}> \\
& E_{n, 0, \nu}^{(2, b)} \equiv<\Psi_{n, 0}^{(0)}\left|-\frac{2 i \nu}{r^{2}} \partial_{\phi}+2 \pi\right| \nu\left|\delta^{(2)}(\mathbf{r})\right| \Psi_{n, 0, \nu}^{(1)}>.
\end{aligned}
$$

It is easy to realize that both $E_{n, 0, \nu}^{(2, a)}$ and $E_{n, 0, \nu}^{(2, b)}$ are divergent, but one can show that the final result $E_{n, 0, \nu}^{(2)}$ is finite. I illustrate the details of the mechanism of cancellation of the infinities by following a definite calculation: the one for $E_{2,0, \nu}^{(2)} \cdot E_{2,0, \nu}^{(2, a)}$ and $E_{2,0, \nu}^{(2, b)}$ are given by

$$
\begin{aligned}
E_{2,0, \nu}^{(2, a)} & =\left\langle\Psi_{2,0}^{(0)}\left|\frac{\nu^{2}}{r^{2}}\right| \Psi_{2,0}^{(0)}>=\nu^{2} \int_{0}^{\infty} \int_{0}^{2 \pi} d r d \phi \frac{\exp \left(-r^{2}\right)}{\pi r}\left[L_{2}^{0}\left(r^{2}\right)\right]^{2}\right. \\
& =\lim _{\epsilon \rightarrow 0} \int_{\epsilon}^{\infty} d r \nu^{2} \frac{\exp \left(-r^{2}\right)}{r}\left[L_{2}^{0}\left(r^{2}\right)\right]^{2}=\nu^{2} \lim _{\epsilon \rightarrow 0}\left[-2 \ln (\epsilon)-\gamma-\frac{3}{2}\right],
\end{aligned}
$$

and

$$
\begin{aligned}
E_{2,0, \nu}^{(2, b)} & =\left\langle\Psi_{2,0}^{(0)}\left|-\frac{2 i \nu}{r^{2}} \partial_{\phi}+2 \pi\right| \nu\left|\delta^{(2)}(\mathbf{r})\right| \Psi_{2,0}^{(1)}\right\rangle=\left\langle\Psi_{2,0}^{(0)}|2 \pi| \nu\left|\delta^{(2)}(\mathbf{r})\right| \Psi_{2,0}^{(1)}\right\rangle \\
& =2 \nu^{2} \int_{-\infty}^{\infty} \int_{-\infty}^{\infty} d r_{x} d r_{y} \delta^{(2)}(\mathbf{r}) e^{-r^{2}} L_{2}^{0}\left(r^{2}\right)\left[\frac{3}{2}-r^{2}+\frac{1}{4}(2 \gamma-3+4 \ln (r)) L_{2}^{0}\left(r^{2}\right)\right] \\
& =\nu^{2} \lim _{\epsilon \rightarrow 0}\left[2 \ln (\epsilon)+\frac{3}{2}+\gamma\right] .
\end{aligned}
$$

Note that I introduced a cut-off $\epsilon$ (which will be ultimately removed by taking the limit $\epsilon \rightarrow 0$ ) in order to see the cancellation of infinities and evaluate the left-over 
finite result. In general a similar cut-off must be introduced in all the divergent matrix elements of $r^{-2}$ and $\delta^{(2)}(\mathbf{r})$ by using

$$
\begin{gathered}
\int_{-\infty}^{\infty} \int_{-\infty}^{\infty} d r_{x} d r_{y} \frac{1}{r^{2}} f\left(r_{x}, r_{y}\right)=\lim _{\epsilon \rightarrow 0} \int_{\epsilon}^{\infty} \int_{0}^{2 \pi} r d r d \phi \frac{1}{r^{2}} f(r \cos \phi, r \sin \phi) \\
\int_{-\infty}^{\infty} \int_{-\infty}^{\infty} d r_{x} d r_{y} \delta^{(2)}(\mathbf{r}) f\left(r_{x}, r_{y}\right)=\lim _{\epsilon \rightarrow 0} f\left(\frac{\epsilon}{\sqrt{2}}, \frac{\epsilon}{\sqrt{2}}\right) .
\end{gathered}
$$

From Eqs.4.4), (4.7), and (4.8) one concludes that $E_{2,0, \nu}^{(2)}=0$, and this is in agreement with Eq.(2.1).

\subsection{Some $N$-anyon energies}

In this subsection I calculate perturbatively from the bosonic end to second order in the statistical parameter $\nu$ and for arbitrary $N$ the eigenenergies of some $N$-anyon in harmonic potential states.

The $\delta$-function regularized Hamiltonian which describes the relative motion of $N$ identical anyons in an harmonic potential is given by

$$
H_{N}^{\delta}=H^{(0)}+\nu H_{L}^{(1)}+|\nu| H_{\delta}^{(1)}+\nu^{2} H^{(2)}
$$

where $H_{0}$ is the relative motion hamiltonian for $\mathrm{N}$ bosons in an harmonic potential, and

$$
\begin{aligned}
H_{L}^{(1)} & \equiv \frac{1}{2} \sum_{m \neq n} \frac{1}{\left|z_{n}-z_{m}\right|^{2}} L_{n, m}, \\
H_{\delta}^{(1)} & \equiv 2 \pi \sum_{m<n} \delta^{(2)}\left(z_{m}-z_{n}\right) \\
H^{(2)} & \equiv \frac{1}{4} \sum_{m \neq n, n \neq k}\left(\frac{1}{\left(z_{n}-z_{m}\right)\left(z_{n}^{*}-z_{k}^{*}\right)}+h . c .\right) ; \\
L_{n, m} & \equiv\left(z_{n}-z_{m}\right)\left(\frac{\partial}{\partial z_{n}}-\frac{\partial}{\partial z_{m}}\right)-\left(z_{n}^{*}-z_{m}^{*}\right)\left(\frac{\partial}{\partial z_{n}^{*}}-\frac{\partial}{\partial z_{m}^{*}}\right) .
\end{aligned}
$$

I am using the conventional notation $z_{n} \equiv r_{n}^{1}+i r_{n}^{2}, z_{n}^{*} \equiv r_{n}^{1}-i r_{n}^{2}$. The center of mass motion is simply a free motion, which I ignore.

The $N$-anyon states whose energies I evaluate perturbatively are the ones which correspond, in the limit $\nu \rightarrow 0$, to the following bosonic states

$$
\mid N, \Omega>\equiv \frac{1}{\sqrt{\pi^{N-1}}} \exp \left[\sum_{n=1}^{N-1}\left|u_{n}\left(\left\{z_{i}\right\}\right)\right|^{2}\right]
$$




$$
\begin{gathered}
\mid N,+2>\equiv \frac{1}{\sqrt{2(N-1) \pi^{N-1}}}\left(\sum_{n=1}^{N-1} u_{n}^{2}\left(\left\{z_{i}\right\}\right)\right) \exp \left[\sum_{n=1}^{N-1}\left|u_{n}\left(\left\{z_{i}\right\}\right)\right|^{2}\right] \\
|N,-2>\equiv| N,+2>^{*}
\end{gathered}
$$

where $u_{n}\left(\left\{z_{i}\right\}\right) \equiv\left(z_{1}+z_{2}+\ldots+z_{n}-n z_{n+1}\right) / \sqrt{n(n+1)}$.

$\mid N, \Omega>$ is the $N$-boson ground state, and the states $\mid N, \pm 2>$ are in the first excited bosonic energy level and have angular momentum \pm 2 .

It is easy to verify that $E_{N, \Omega}^{(1,2)}(\nu)=E_{N, \Omega}^{(1,2)}(-\nu), E_{N,+2}^{(1)}(\nu)=-E_{N,-2}^{(1)}(-\nu)$, and $E_{N,+2}^{(2)}(\nu)=$ $E_{N,-2}^{(2)}(-\nu)$; therefore, I can limit the calculations to the case $\nu>0$ without any loss of generality.

The first order energies can be easily calculated; they are given by

$$
\begin{gathered}
E_{N, \Omega}^{(1)}=<N, \Omega\left|\nu H_{L}^{(1)}+\right| \nu\left|H_{\delta}^{(1)}\right| N, \Omega> \\
=\frac{N(N-1)}{2}<N, \Omega\left|2 \pi \nu \delta^{(2)}\left(z_{1}-z_{2}\right)\right| N, \Omega> \\
=\frac{N(N-1)}{2} \nu \\
E_{N, \pm 2}^{(1)}=<N, \pm 2\left|\nu H_{L}^{(1)}+\right| \nu\left|H_{\delta}^{(1)}\right| N, \pm 2> \\
=\frac{N(N-1)}{2}<N, \pm 2\left|\nu \frac{L_{12}}{\left|z_{1}-z_{2}\right|^{2}}+2 \pi \nu \delta^{(2)}\left(z_{1}-z_{2}\right)\right| N, \pm 2> \\
=\frac{N(N-2)}{2} \nu \pm \frac{N}{2} \nu .
\end{gathered}
$$

Concerning the evaluation of the second order energies, let me start by noticing that from (4.11) and (4.12) it follows that

$$
\begin{aligned}
E_{\Psi^{(0)}}^{(2)} & =<\Psi^{(0)}\left|\nu^{2} H^{(2)}\right| \Psi^{(0)}>+E_{\Psi^{(0)} ; L}^{(2)}+E_{\Psi^{(0)} ; \delta}^{(2)} \\
E_{\Psi^{(0)} ; L}^{(2)} & \equiv \sum_{\mid m>\notin D} \frac{<\Psi^{(0)}\left|\nu H_{L}^{(1)}+\right| \nu\left|H_{\delta}^{(1)}\right| m><m\left|\nu H_{L}^{(1)}\right| \Psi^{(0)}>}{E^{(0)}-E_{m}^{(0)}} \\
E_{\Psi^{(0)} ; \delta}^{(2)} & \equiv \sum_{\mid m>\notin D} \frac{<\Psi^{(0)}\left|\nu H_{L}^{(1)}+\right| \nu\left|H_{\delta}^{(1)}\right| m><m|| \nu\left|H_{\delta}^{(1)}\right| \Psi^{(0)}>}{E^{(0)}-E_{m}^{(0)}} .
\end{aligned}
$$

Using the symmetries of $H^{(2)}$ and of the unperturbed wave functions (4.13), (4.14), and (4.15), one easily obtains

$$
\begin{aligned}
<N, \Omega\left|\nu^{2} H^{(2)}\right| N, \Omega>= & \frac{\nu^{2}}{4} N(N-1)\left[2(N-2) \ln \left(\frac{4}{3}\right)-\gamma\right] \\
& -\lim _{\epsilon \rightarrow 0}\left[\frac{\nu^{2}}{4} N(N-1) \ln (\epsilon)\right],
\end{aligned}
$$




$$
\begin{aligned}
<N, \pm 2\left|\nu^{2} H^{(2)}\right| N, \pm 2>= & \frac{\nu^{2}}{8} N\left[9-4 N+4(N+1)(N-2) \ln \left(\frac{4}{3}\right)-2 \gamma(N-2)\right] \\
& -\lim _{\epsilon \rightarrow 0}\left[\frac{\nu^{2}}{4} N(N-2) \ln (\epsilon)\right]
\end{aligned}
$$

where the cut-off $\epsilon$ has been introduced using (4.9).

For the states (4.13), (4.14), and (4.15) the evaluation of $E_{\Psi^{(0)} ; L}^{(2)}$ and $E_{\Psi^{(0)} ; \delta}^{(2)}($ which usually is only possible numerically) is relevantly simplified by the following results

$$
\begin{aligned}
\frac{1}{\left|z_{1}-z_{2}\right|^{2}} L_{12} \mid N, \pm 2> & =\frac{1}{4}\left(\left[C, H^{(0)}\right]-\pi \delta^{(2)}\left(\frac{z_{1}-z_{2}}{\sqrt{2}}\right)+1\right) L_{12} \mid N, \pm 2> \\
<m\left|\left[C, H^{(0)}\right]\right| N, \Omega( \pm 2)> & =<m\left|\pi \delta^{(2)}\left(\frac{z_{1}-z_{2}}{\sqrt{2}}\right)\right| N, \Omega( \pm 2)> \\
H^{(0)} L_{12} \mid N, \pm 2> & =E_{N, \pm}^{(0)} L_{12} \mid N, \pm 2> \\
L_{12} \mid N, \Omega> & =0 .
\end{aligned}
$$

where $C=\frac{1}{2}\left(\ln \left(\frac{\left|z_{1}-z_{2}\right|^{2}}{2}\right)+\gamma-1\right)$.

Using the properties (4.22) one finds

$$
\begin{aligned}
& E_{N, \Omega ; L}^{(2)}=0, \\
& E_{N, \Omega ; \delta}^{(2)}=\frac{\nu}{2}\left(N^{2}-N\right)\left[<N, \Omega \mid\left(\nu H_{L}^{(1)}+|\nu| H_{\delta}^{(1)} C\left|N, \Omega>-E_{N, \Omega}^{(1)}<N, \Omega\right| C \mid N, \Omega>\right]\right. \\
& =\lim _{\epsilon \rightarrow 0}\left[\frac{\nu^{2}}{4} N(N-1)\left(\ln (\epsilon)+\gamma-2(N-2) \ln \left(\frac{4}{3}\right)\right)\right], \\
& E_{N, \pm 2 ; L}^{(2)}=\frac{\nu}{8}\left(N^{2}-N\right)\left[<N, \pm 2\left|\left(\nu H_{L}^{(1)}+|\nu| H_{\delta}^{(1)}\right) C L_{12}\right| N, \pm 2>\right. \\
& \left.-E_{N, \pm 2}^{(1)}<N, \pm 2\left|C L_{12}\right| N, \pm 2>\right] \\
& = \pm \frac{3}{8} \nu^{2} N(N-2) \ln \left(\frac{3}{4}\right)+\frac{\nu^{2}}{16} N\left(5 N-12-18(N-2) \ln \left(\frac{4}{3}\right)\right) \\
& E_{N, \pm 2 ; \delta}^{(2)}=\frac{\nu}{2}\left(N^{2}-N\right)\left[<N, \pm 2\left|\left(\nu H_{L}^{(1)}+|\nu| H_{\delta}^{(1)}\right) C \frac{\sum_{n=2}^{N-1} u_{n}^{2}\left(\left\{z_{i}\right\}\right)}{\sum_{k=1}^{N-1} u_{k}^{2}\left(\left\{z_{i}\right\}\right)}\right| N, \pm 2>\right. \\
& \left.-E_{N, \pm 2}^{(1)}<N, \pm 2\left|C \frac{\sum_{n=2}^{N-1} u_{n}^{2}\left(\left\{z_{i}\right\}\right)}{\sum_{k=1}^{N-1} u_{k}^{2}\left(\left\{z_{i}\right\}\right)}\right| N, \pm 2>\right] \\
& =\frac{\nu^{2}}{16} N \lim _{\epsilon \rightarrow 0}\left[3 N-6+4(N-2)(\gamma+\ln (\epsilon))+\left(38 N-44-8 N^{2}\right) \ln \left(\frac{4}{3}\right)\right] \\
& \pm \frac{3}{8} \nu^{2} N(N-2) \ln \left(\frac{3}{4}\right) \text {. }
\end{aligned}
$$


Again I introduced the cut-off $\epsilon$ using (4.10).

Combining these results with (4.16), (4.17), (4.18), and (4.21) one obtains the following final results:

$$
\begin{gathered}
E_{N, \Omega}^{(1)}+E_{N, \Omega}^{(2)}=\frac{N(N-1)}{2} \nu, \\
E_{N,+2}^{(1)}+E_{N,+2}^{(2)}=\frac{N(N-1)}{2} \nu, \\
E_{N,-2}^{(1)}+E_{N,-2}^{(2)}=\frac{N(N-3)}{2} \nu+\ln \left(\frac{4}{3}\right) \frac{3 N(N-2)}{2} \nu^{2} .
\end{gathered}
$$

$E_{N, \Omega}$ and $E_{N,+2}$ are among the exactly known eigenenergies of the $N$-anyon in harmonic potential problem (see Ref.[9]), and they are in perfect agreement with (4.27) and (4.28). $E_{N,-2}$ is not known exactly, but, for the special cases $N=3$ and $N=4$, $E_{N,-2}^{(1)}+E_{N,-2}^{(2)}$ has been calculated numerically in Refs. 17, 18] and perturbatively, using the regularization method II, in Refs. 13, 14; Eq.(4.29), for the corresponding values of $N$, is in agreement with those results.

\subsection{Additional 3-anyon energies}

The N-anyon eigenenergies calculated to second order in $\nu$ in the preceding subsection and in Ref.[16] all concerned bosonic end states with particularly symmetric wave functions (besides the bosonic symmetry under exchange of the particle indices, they were also symmetric under permutations of the functions $u_{i}$ ). This special form of the bosonic end wave functions leads to simplifications that were exploited in obtaining the results for arbitrary $N$. In order to perform a more general test of the $\delta$-function regularization (i.e. show that the agreement with the exact results found in the preceding subsection is not a consequence of the special form of the wave functions considered), I now calculate to second order in $\nu$ the eigenenergies of the two 3-anyon states whose bosonic end wave functions are given by

$$
\begin{gathered}
\mid 3,+3>\equiv \frac{1}{\sqrt{24 \pi^{3}}} u_{2}\left(\left\{z_{i}\right\}\right)\left(3\left[u_{1}\left(\left\{z_{i}\right\}\right)\right]^{2}-\left[u_{2}\left(\left\{z_{i}\right\}\right)\right]^{2}\right) \exp \left[\left|u_{1}\left(\left\{z_{i}\right\}\right)\right|^{2}+\left|u_{2}\left(\left\{z_{i}\right\}\right)\right|^{2}\right] \\
|3,-3>\equiv| 3,+3>^{*}
\end{gathered}
$$

(Notice that $\mid 3, \pm 3>$ are not symmetric under permutations of the functions $u_{i}$.) The states $\mid 3, \pm 3>$ are in the second excited energy level and have angular momentum \pm 3 .

Concerning the first order energies $E_{3, \pm 3}^{(1)}$ one easily finds that

$$
E_{3, \pm 3}^{(1)}=\left(\frac{3}{4} \pm \frac{9}{4}\right) \nu
$$

It is also easy to show that the states $\mid 3, \pm 3>$ satisfy relations of the type (4.22), and this allows to evaluate the second order energies $E_{3, \pm 3}^{(2)}$ analytically. Following a 
procedure completely analogous to the one discussed in the preceding subsection, I find that

$$
\begin{aligned}
E_{3, \pm 3}^{(2)} & =<3, \pm 3\left|\nu^{2} H^{(2)}\right| 3, \pm 3>+E_{3, \pm 3 ; L}^{(2)}+E_{3, \pm 3 ; \delta}^{(2)} \\
<3, \pm 3\left|\nu^{2} H^{(2)}\right| 3, \pm 3> & =\nu^{2}\left(2+\frac{9}{16}-6 \ln \left(\frac{4}{3}\right)\right) \\
E_{3, \pm 3 ; L}^{(2)} & =\frac{\nu^{2}}{64}\left(486 \ln \left(\frac{4}{3}\right)-189\right) \pm \frac{\nu^{2}}{32}\left(9-27 \ln \left(\frac{4}{3}\right)\right) \\
E_{3, \pm 3 ; \delta}^{(2)} & =\frac{\nu^{2}}{64}\left(6 \ln \left(\frac{4}{3}\right)-11\right) \pm \frac{\nu^{2}}{32}\left(9-27 \ln \left(\frac{4}{3}\right)\right)
\end{aligned}
$$

From Eqs.(4.31) and (4.32) one concludes that

$$
\begin{aligned}
& E_{3,+3}^{(1)}+E_{3,+3}^{(2)}=3 \nu \\
& E_{3,-3}^{(1)}+E_{3,-3}^{(2)}=-\frac{3}{2} \nu+\frac{9}{8}\left(3 \ln \left(\frac{4}{3}\right)-1\right) \nu^{2} .
\end{aligned}
$$

As expected the $\delta$-function regularization result for $E_{3,+3}^{(1)}+E_{3,+3}^{(2)}$ is in perfect agreement with the corresponding exact result obtained in Ref.[7]. $E_{3,-3}^{(1)}+E_{3,-3}^{(2)}$ is not known exactly, but it was calculated numerically in Refs. [17, 18] and perturbatively, using the regularization method II, in Ref. 113]; Eq.(4.34) agrees with those results.

\section{Summary and Conclusion}

In the first part of the paper I have examined the three bosonic end perturbative approaches that have been used in the study of the anyon spectra. I find that the $\delta$-function regularization has the most transparent physical interpretation, and this is very important if we want to be confident in using bosonic end perturbation theory in the study of the presently unknown portion of the anyon spectra. Moreover, as illustrated by the calculation for arbitrary number of anyons in Sec.IV, in some instances properties of the $\delta$-function regularized Hamiltonian can be exploited to achieve significant simplification.

I have also presented evidence in support of the equivalence of the regularization method I and the $\delta$-function regularization (which was already argued for in Ref. [21]). This equivalence is confirmed by the agreement between all the results obtained with these two methods. The regularization method I has a less insightful physical interpretation, but the fact that the corresponding Hamiltonian only includes 2-body interactions can be useful in some calculations.

Concerning the regularization method II, I found that it essentially corresponds to a less efficient (and less motivated) way to implement the same program of the $\delta$-function regularization. As indicated by calculations presented in Sec.IV, the results of the regularization method II are reliable at least to second order in $\nu$. In particular, using the $\delta$-function regularization, I confirmed the important result for $E_{3,-3}$ obtained in Ref.[13], which, combined with results of Ref. [9], allows an approximate analytic description of the 3 -anyon in harmonic oscillator potential ground state energy for all values of $\nu$. These calculations of $E_{3,-3}$ also represent the only analytic 
evidence for the existence of anyonic wave functions with energies non-linearly dependent on $\nu$ that smoothly interpolate from the bosonic to the fermionic limit 28]. Now that the result of Ref. 13 has been confirmed using a more transparent procedure, there is additional motivation for the use of the analysis presented in Refs. 9, 13 as a guide in the search for additional exact solutions to the $N$-anyon $(N>2)$ problem.

The calculation of $E_{N,-2}^{(1)}+E_{N,-2}^{(2)}$ discussed in detail in Sec.IV is an important result of the $\delta$-function regularization; in fact, it gives the only direct evidence [16] of the fact that there are many-anyon eigenenergies with non-linear dependence on the statistical parameter $\nu$. I also want to emphasize again [13, 14, 16] that the presence of the interesting factor $\ln (4 / 3)$ in the non-vanishing second order contributions to the energies (see for example Eqs.(4.29) and (4.34)) could suggest Ansätze (like the one proposed in Refs. [13, 14]) that simplify the search for new exact anyon eigensolutions.

Finally, I want to mention a possible area of investigation which might be motivated by the results discussed in this paper, but is not aimed at the study of anyon physics. As indicated in Sec.III, the $\delta$-function regularization can be cast precisely in the form of a renormalization procedure [20, 26] like the ones customary in the field theoretical framework. However, whereas in (relativistic) field theory usually interesting results can only be obtained in perturbation theory, in anyon quantum mechanics in several cases one has available (at least some of) the exact solutions. In anyon quantum mechanics it is therefore sometime possible to compare the (renormalizationrequiring) perturbative results with the exact results. These comparisons might lead to some insight in the physics behind the renormalization and regularization procedures necessary in field theory. Work on this subject is now in progress 29.

\section{Acknowledgements}

I have benefitted from conversations with D. Bak, R. Jackiw, and C. Manuel; these I gratefully acknowledge. 


\section{References}

[1] J. M. Leinaas and J. Myrheim, Nuovo Cimento B37 (1977) 1; G. A. Goldin, R. Menikoff, and D. H. Sharp, J. Math. Phys. 21 (1980) 650; 22 (1981) 1664; F. Wilczek, Phys. Rev. Lett. 48 (1982) 1144; 49 (1982) 957 . Also see: F. Wilczek, Fractional Statistics and Anyon Superconductivity, (World Scientific, 1990).

[2] R. Jackiw and S.-Y. Pi, Phys. Rev. D42 (1990) 3500.

[3] R. B. Laughlin, Phys. Rev. Lett. 50 (1983) 1395; B. I. Halperin, Phys. Rev. Lett. 52 (1984) 1583.

[4] R. Jackiw, Ann. Phys. 129 (1980) 183; Ann. Phys. 201 (1990) 83.

[5] M. Sporre, J. J. M. Verbaarschot, and I. Zahed, Nucl. Phys. B389 (1993) 645.

[6] D. Sen, Phys. Rev. D45 (1992) 1846; D. Sen and R. Chitra, Phys. Rev. B45 (1992) 881.

[7] Y. S. Wu, Phys. Rev. Lett. 53 (1984) 111.

[8] G. V. Dunne, A. Lerda, and C. A. Trugenberger, Mod. Phys. Lett. A6 (1991) 2819; G. V. Dunne, A. Lerda, S. Sciuto, and C. A. Trugenberger, Nucl. Phys. B370 (1992) 601; Chihong Chou, Phys. Lett. A155 (1991) 245; A. Polychronakos, Phys.Lett. B264 (1991) 362; K. H. Cho and C. Rim, Ann. Phys. 213 (1992) 295; S. Mashkevich, Int. J. Mod. Phys. A7 (1992) 7931.

[9] C. Chou, Phys. Rev. D44 (1991) 2533; Erratum-ibid. D45 (1992) 1433.

[10] A. Khare and J. McCabe, Phys. Lett. B269 (1991) 330.

[11] J. McCabe and S. Ouvry, Phys. Lett. B260 (1991) 113; A. Comtet, J. McCabe, and S. Ouvry, Phys. Lett. B260 (1991) 372; A. Dasnieres de Veigy and S. Ouvry, Phys. Lett. B291 (1992) 130; Nucl. Phys. B388 (1992) 715.

[12] D. Sen, Nucl. Phys. B360 (1991) 397.

[13] C. Chou, L. Hua, and G. Amelino-Camelia, Phys. Lett. B286 (1992) 329.

[14] G. Amelino-Camelia, Phys. Lett. B299 (1992) 83.

[15] J.Y. Kim, O.J. Kwon, Y.S. Myung, and S.H. Yi, Phys. Rev. D48 (1993) 4839.

[16] G. Amelino-Camelia, Phys. Lett. B326 (1994) 282.

[17] M. Sporre, J. J. M. Verbaarschot, and I. Zahed, Phys. Rev. Lett. 67 (1991) 1813; SUNY-NTG-91/40 (1991).

[18] M. V. N. Murthy, J. Law, M. Brack, and R. K. Bhaduri, Phys. Rev. Lett. 67 (1991) 1817.

[19] G. Amelino-Camelia, Phys. Lett. B286 (1992) 97.

[20] C. Manuel and R. Tarrach, UB-ECM-PF19/93 (to appear in Phys.Lett. B). The reader should notice that in this paper the same regularization procedure of Ref. [16] is used, even though, rather than the anyon spectra, the (closely related) Aharonov-Bhom scattering problem is studied.

[21] S. Ouvry, IPNO/TH93-71 (1993). 
[22] G. Lozano, Phys. Lett. B283 (1992) 70; M.A. Valle Basagoiti, Phys.Lett. B306 (1993) 307; D.Z. Freedman, G. Lozano, and N. Rius, Phys. Rev. D49 (1994) 1054; O. Bergman and G. Lozano, Ann. Phys. 229 (1994) 416.

[23] M. Bourdeau and R.D. Sorkin, Phys. Rev. D45 (1992) 687.

[24] C. Manuel and R. Tarrach, Phys. Lett. B268 (1991) 222.

[25] D. Bak, O. Bergman, MIT-CTP-2283 (1994).

[26] R. Jackiw, in M.A.B. Bèg memorial volume, A. Ali and P. Hoodbhoy eds. (World Scientific, Singapore, 1991).

[27] P. de Sousa Gerbert, Phys. Rev. D40 (1989) 1346; D. Caenepeel and R. MacKenzie, UdeM-LNP-TH-94-188 (1994); D.K. Park, HEPTH-9405009 (1994).

[28] G. Amelino-Camelia and L. Hua, Phys. Rev. Lett. 69 (1992) 2875; K. H. Cho and C. Rim, Phys. Rev. Lett. 69 (1992) 2877.

[29] G. Amelino-Camelia and D. Bak, in preparation. 